\title{
OVERCOMING THE NEGATIVE FREQUENCIES - INSTANTANEOUS FREQUENCY AND AMPLITUDE ESTIMATION USING OSCULATING CIRCLE METHOD
}

\author{
Ming-Kuang Hsu \\ Center for General Education, Technology and Science Institute of Northern Taiwan, Taipei, Taiwan, R.O.C., \\ hsu.ming.kuang@gmail.com \\ Jiun-Chyuan Sheu \\ Wells Fargo Bank, San Francisco, CA, USA. \\ Cesar Hsue \\ Parker Hannifin Corporation, Irvine, CA, USA.
}

Follow this and additional works at: https://jmstt.ntou.edu.tw/journal

Part of the Engineering Commons

\footnotetext{
Recommended Citation

Hsu, Ming-Kuang; Sheu, Jiun-Chyuan; and Hsue, Cesar (2011) "OVERCOMING THE NEGATIVE FREQUENCIES INSTANTANEOUS FREQUENCY AND AMPLITUDE ESTIMATION USING OSCULATING CIRCLE METHOD," Journal of Marine Science and Technology. Vol. 19: Iss. 5, Article 7.

DOI: 10.51400/2709-6998.2165

Available at: https://jmstt.ntou.edu.tw/journal/vol19/iss5/7

This Research Article is brought to you for free and open access by Journal of Marine Science and Technology. It has been accepted for inclusion in Journal of Marine Science and Technology by an authorized editor of Journal of Marine Science and Technology.
} 


\section{OVERCOMING THE NEGATIVE FREQUENCIES - INSTANTANEOUS FREQUENCY AND AMPLITUDE ESTIMATION USING OSCULATING CIRCLE METHOD}

\section{Acknowledgements}

The first author would like to thank Academician N.E. Huang who introduced the EMD method when the first author visited NASA in 1998, and subsequently gave the first author many valuable comments on this new OC method. The first author also would like to thank Dr. Antony K. Liu for inviting the first author to conduct research at NASA in 1998 where the first author originated the concept of the OC method. The financial support from ONR and National Taiwan Ocean University (NTOU) that enabled the first author to visit NASA is greatly appreciated. The continuous support from National Science Council (for 2008 project no. is NSC 97-2611-M149-001), and support from Technology and Science Institute of Northern Taiwan (TSINT) is also greatly appreciated. TSINT has filed a patent application for the OC method. 


\title{
OVERCOMING THE NEGATIVE FREQUENCIES - INSTANTANEOUS FREQUENCY AND AMPLITUDE ESTIMATION USING OSCULATING CIRCLE METHOD
}

\author{
Ming-Kuang Hsu*, Jiun-Chyuan Sheu**, and Cesar Hsue***
}

Key words: Hilbert transforms, osculating circle, instantaneous frequency, unwrapped phase.

\begin{abstract}
There are many methods able to extract the instantaneous frequencies from a time series for practical applications. Gabor's method is one of the most popular and simple methods. For wide band time series, Gabor's method may produce negative frequencies. The limitation of Gabor's method is that, in order to avoid resulting with negative frequencies, it can only be applied to time series with mono component and near zero mean. In this paper, a new method that does not generate negative frequencies, the Osculating Circle (OC) Method, is presented. The OC method is based on Gabor's method but with modified unwrapped phase calculation. This new method can provide accurate instantaneous frequency (IF) and instantaneous amplitude (IA) calculation with only positive frequency results. This paper is focused on overcoming the negative frequency encountered in traditional Garbo's method. Although, other important topics such as the noise are not discussed in detail in this paper.
\end{abstract}

\section{INTRODUCTION}

For non-stationary signals whose spectral contents vary with time, the frequency at a particular instance can be characterized by its instantaneous frequency (IF). In many applications such as communication systems, bio-medical engineering, failure analysis, the IF can depict important physical parameters of the signals. It is, therefore, critical to have effective methods for IF calculation. Using Gabor's method for IF calculation is limited because the results may include

Paper submitted 03/24/09; revised 04/18/10; accepted 05/27/10. Author for correspondence: Ming-Kuang Hsu (e-mail: hsu.ming.kuang@gmail.com).

*Center for General Education, Technology and Science Institute of Northern Taiwan, Taipei, Taiwan, R.O.C.

**Wells Fargo Bank, San Francisco, CA, USA.

***Parker Hannifin Corporation, Irvine, CA, USA. negative frequencies. In this paper, we introduce a new method, the Osculating Circle (OC) Method, that can overcome the limitation of negative frequencies when Gabor's method is used.

\section{THE INSTANTANEOUS FREQUENCY OBTAINED THROUGH HILBERT TRANSFORM}

Many methods had been invented and used to estimate instantaneous frequency. These methods include Short-Time Fourier Transform, Wigner Distribution [1, 2], Gabor's method $[3,4,6]$ Wavelet Transform, and EMD [7]. Among these methods, Gabor's method [4] that uses Hilbert Transform (HT) is the simplest. In this section, we will discuss the Hilbert transfer and issues with negative frequencies.

\section{Hilbert Transform (HT)}

For an arbitrary time series, $x(t)$, we can derive its HT, $y(t)$, as

$$
y(t)=\frac{-1}{\pi} P \int_{-\infty}^{\infty} \frac{x(\tau)}{t-\tau} d \tau
$$

where $P$ is the Cauchy principal value [6]. With this definition, $x(t)$ and $y(t)$ form a complex conjugate pair and we have an analytic signal, $z(t)$, as

$$
z(t)=x(t)+i y(t)=a(t) e^{i \theta(t)}
$$

where

$$
a(t)=\left[x^{2}(t)+y^{2}(t)\right]^{1 / 2}
$$

and

$$
\theta(t)=\tan ^{-1}\left(\frac{y(t)}{x(t)}\right)
$$




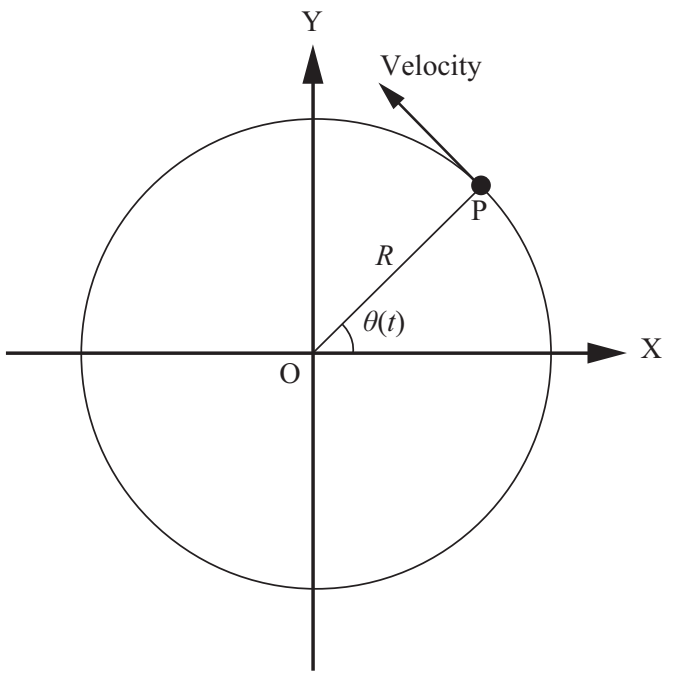

(a)

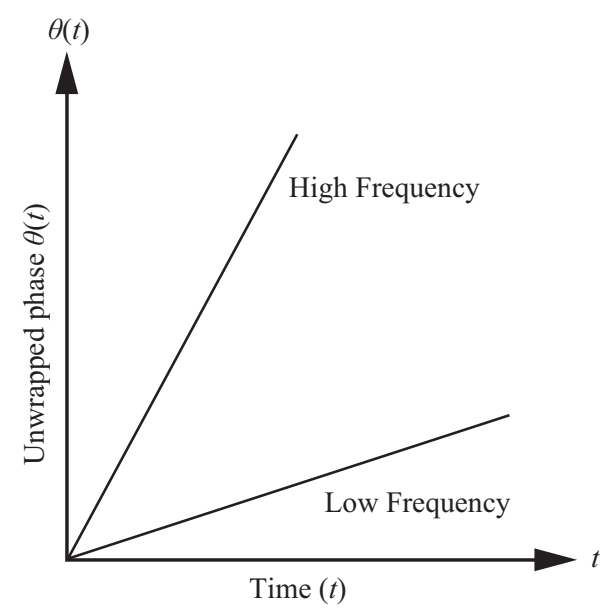

(b)

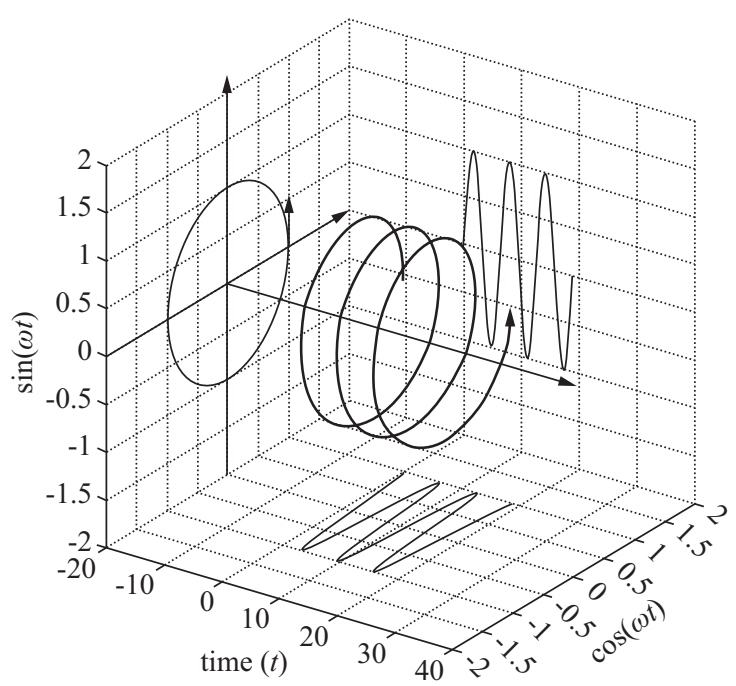

(c)

Fig. 1. (a) A cosine function (with a global mean of 0 ) and its HT form a circle in $x-y$ plane. (b) The unwrapped phase vs. time. Higher angular speed would have steeper slope and vice versa. (c) A three dimensional view of $\cos (\omega t)$; its HT, $\sin (\omega t)$; and time.

\section{Instantaneous Frequency for a Simple Harmonic Function}

Assume a time series, $x(t)$, is a cosine function with a global mean of 0 . The HT of $x(t)$ is a sine function. These two time series form a circular trajectory in $x-y$ plane (Fig. 1(a)). We can imagine this as a particle, $\mathrm{P}$, moving counterclockwise along a circle with a radius, $\mathrm{R}$, and a constant angular speed. The position of the particle can be expressed as the unwrapped phase vs. time (Fig. 1(b)) and the slope of the unwrapped phase-time plot is the frequency of the particle moving along the circle. A steeper slope corresponds to a higher frequency and vice versa. When the angular speed of the particle varies with time, the slope of the unwrapped phase will change with time as well. Fig. 1(c) is the three dimensional view of a time series, $\cos (\omega t)$; its HT, $\sin (\omega t)$; and the spiral trajectory of the particle when time is used as the third axis.

Suppose we define instantaneous frequency as the time derivative of the unwrapped phase of the analytic signal

$$
\Omega(t)=\frac{1}{2 \pi} \frac{d \theta(t)}{d t}
$$

where $\theta(t)$ is unwrapped phase and $\Omega(t)$ is the IF in Hz. In order for IF to be meaningful (i.e., always positive), the slope of the unwrapped phase-time plot has to be always positive. This implies that the time signal must be locally symmetric to the zero mean $[3,6,7]$. Since the majority of real life data does not satisfy this requirement, the Gabor's method often produces results with negative frequencies that are difficult to interpret and use.

\section{Negative Instantaneous Frequency of the Analytic Signal}

Cohen [3] and Hahn [6] noted that, with Gabor's method, the instantaneous frequency of wideband analytic signals may change its signs. An example from their work [6] is presented here.

Let $x(t)$ be the sum of two simple harmonic signals such as

$$
x(t)=\cos (a t / 2)+k \cos a t
$$

where $a$ and $k$ are real constants. The combined signal is showed in Fig. 2 with $a=2 \pi / 512$ and $k=0.8$. Fig. 2(a) shows one cycle of time series $x(t)$. Fig. 2(b) is the HT of $x(t)$. Fig. 2(c) is the trajectory of a particle formed by $x(t)$ and its HT. For a particle moving along the trajectory counterclockwise as shown in Fig. 2(c), the solid line in Fig. 2(d) is the unwrapped phase-time plot. Further, the solid line in Fig. 2(e) is the instantaneous frequency obtained by (5) and the solid line in Fig. 2(f) is the instantaneous amplitude which is the distance from the origin to the particle. Since some portion of the slope is negative (solid line in Fig. 2(d)), the corresponding negative IF is derived as showed by the solid line in Fig. 2(e). The IF of wideband analytic signals will change from positive to 


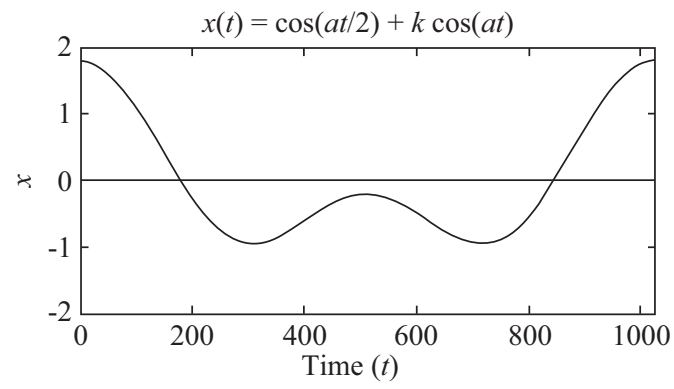

(a)

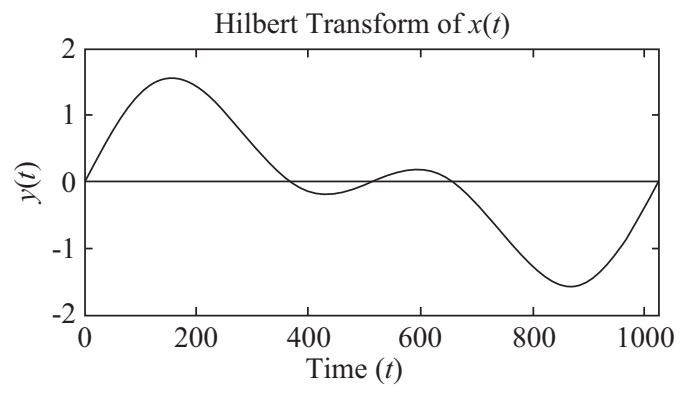

(b)

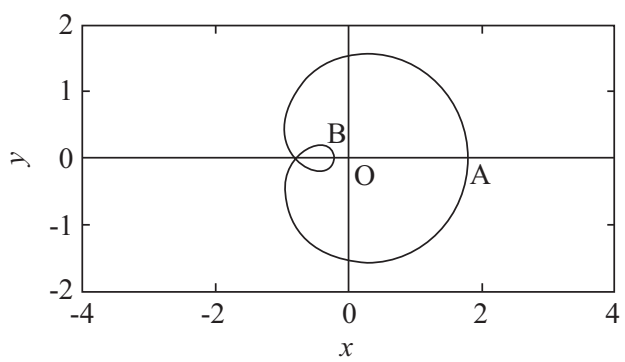

(c)

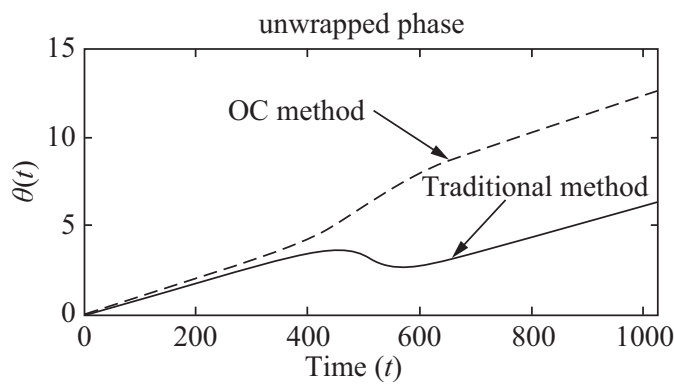

(d)

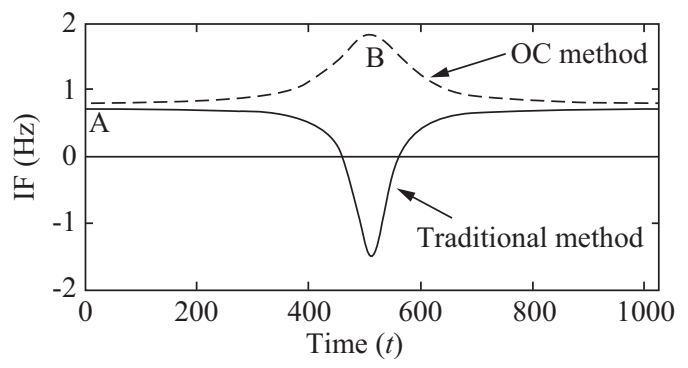

(e)

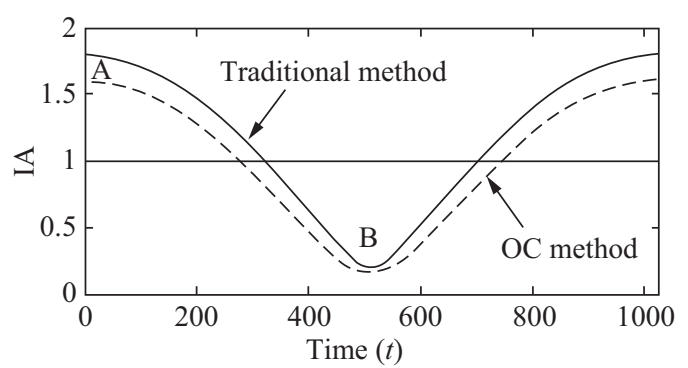

(f)

Fig. 2. (a) $x(t)=\cos (a t / 2)+k \cos a t$ with $k=0.8$ and $a=2 \pi / 512$, (b) HT of $x(t)$, (c) the trace of the particle, (d) unwrapped phase $v s$. time, (e) instantaneous frequency, and (f) instantaneous amplitude. In (d), (e), and (f), the traditional Gabor's method is in solid line and OC Method is in dashed line.

negative when the phasor changes its rotation from counterclockwise to the clockwise.

Under certain circumstances such as a DC offset, even a mono-component time series can result negative instantaneous frequencies [7]. An example from Huang et al. [7] work is presented here. Consider a cosine time series with some DC offset of $d$

$$
x(t)=d+R \cos \omega t
$$

Three time series are shown in Fig. 3(a). The solid line shows no offset $(d=0)$. The dash line shows an offset smaller than the amplitude $(d=0.8$ and $|d| \leq R)$. The dash dot line shows an offset larger than the amplitude $(d=1.5$ and $|d|>R)$ Although the value of $d$ is different in these three cases, their HT are the same $(R \sin \omega t)$ as showed in Fig. 3(b). Their phase plots of $x-y$ (trajectories) are simple circles with the radius $R$ but centered at different location as showed in Fig. 3(c). Their unwrapped phase functions with corresponding $d$ are showed in Fig. 3(d). The IF for corresponding $d$ are shown in Fig. 3(e). The IA with corresponding $d$ are showed in Fig. 3(f).

When there is no offset, $|d|=0$, the center of the circle (trajectory) is at the origin (solid line in Fig. 3(c)). For a particle moving along the circle with a constant angular speed, the slope of unwrapped phase-time plot is a straight line (solid line in Fig. 3(d)). The IF (solid line in Fig. 3(e)) and IA (solid line in Fig. 3(f)) obtained by Gabor's method are constant.

When the offset is smaller than the amplitude, $|d| \leq R$, the origin will be located inside the circular trajectory but not at the center (dash line in Fig. 3(c)). Although the particle moves along the circle (trajectory) with a constant angular speed, the slope of unwrapped phase varies with time. Since the slopes of unwrapped phase are always positive (dash line in Fig. 3(d)), the IF derived by (5) is also always positive except its value varied with time (dash line in Fig. 3(e)). The Gabor's IA, which is defined as the distance between the origin and the particle, is showed as dash line in Fig. 3(f).

When the distance between the origin and the particle is 


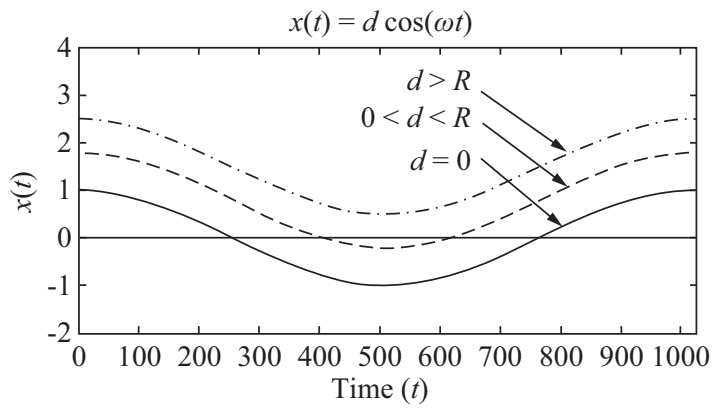

(a)

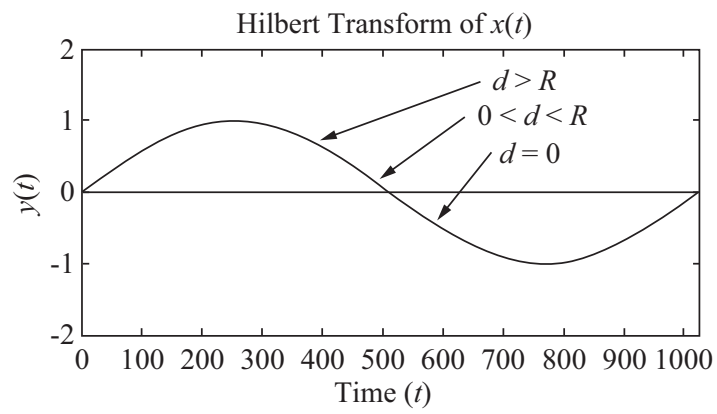

(b)

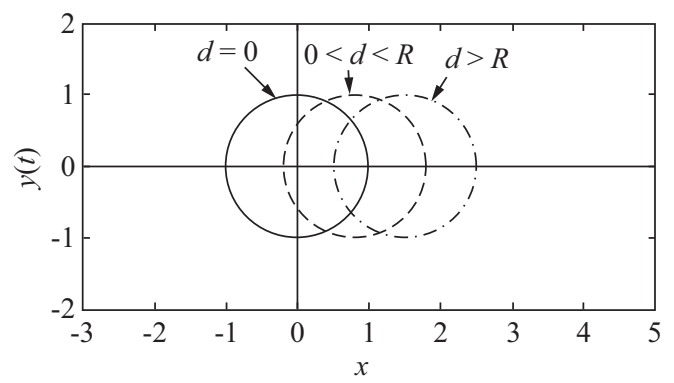

(c)

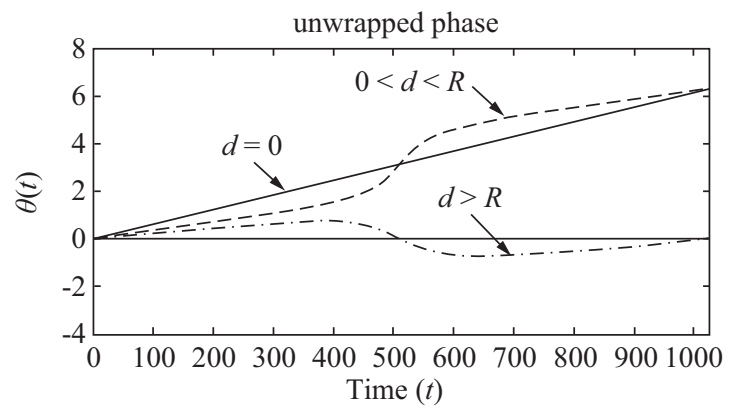

(d)

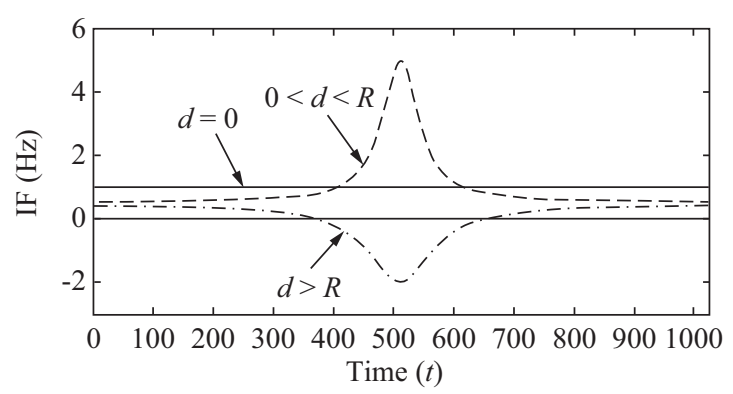

(e)

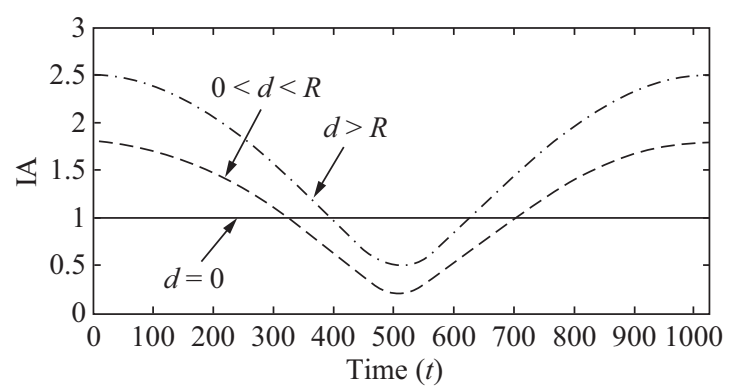

(f)

Fig. 3. (a) $x(t)=d+R \cos \omega t$, (b) HT of $x(t)$, (c) the path of the particle, (d) unwrapped phase vs. time, (e) instantaneous frequency, and (f) instantaneous amplitude, where $R=1 ; d=0$ (solid line), $d=0.8$ (dash line), and $d=1.5$ (dash dot line). When $|d| \geq R$, the unwrapped phase may have negative slopes with corresponding negative frequencies.

large, IA will be large and vice versa. In the case when $|d|>R$, the origin is outside of the circular trajectory (dash dot line in Fig. 3(c)). Like the previous case, even if a particle is moving with a constant angular speed, the slope of unwrapped phase-time in this case is not a constant (dash dot line in Fig. $3(d)$ ). In the region where the slope of unwrapped phase function is negative, the corresponding IF to that region will be negative (dash dot line in Fig. 3(e)). The IA are showed in Fig. 3(f) as the dash dot line.

Intuitively, for a particle with a circular trajectory and a constant angular speed, the frequency (IF) and the amplitude (IA) should be a constant at any instant regardless the DC offset. However, in the cases presented above, both IF and IA are not constant with non-zero offsets. The example illustrated the fact that using Gabor's method, the IF for a simple harmonic signal, such as a cosine function, can be accurately depicted only for functions that are symmetric locally with zero mean. Any DC offset can cause inaccurate IF and IA measurements. This limitation is detrimental to most practical applications and needs to be removed [7]. One approach to overcome this restriction is to decompose the data in such a way that the instantaneous frequency can be properly calculated. Huang et al. [7] developed the Empirical Mode Decomposition Method (EMD Method) that decomposes a signal into a series of zero mean Intrinsic Mode Functions (IMFs). For each IMF, the instantaneous frequency can be defined everywhere.

In the following sections, we will present a simple approach to solve the Gabor's negative frequency limitation.

\section{Using the Osculating Circle Method to Derive the Instantaneous Frequency and Corresponding Amplitude}

The basis of the osculating circle method is similar to the traditional Gabor's method but with modified definitions of unwrapped phase and the time dependent amplitude (IA). In 


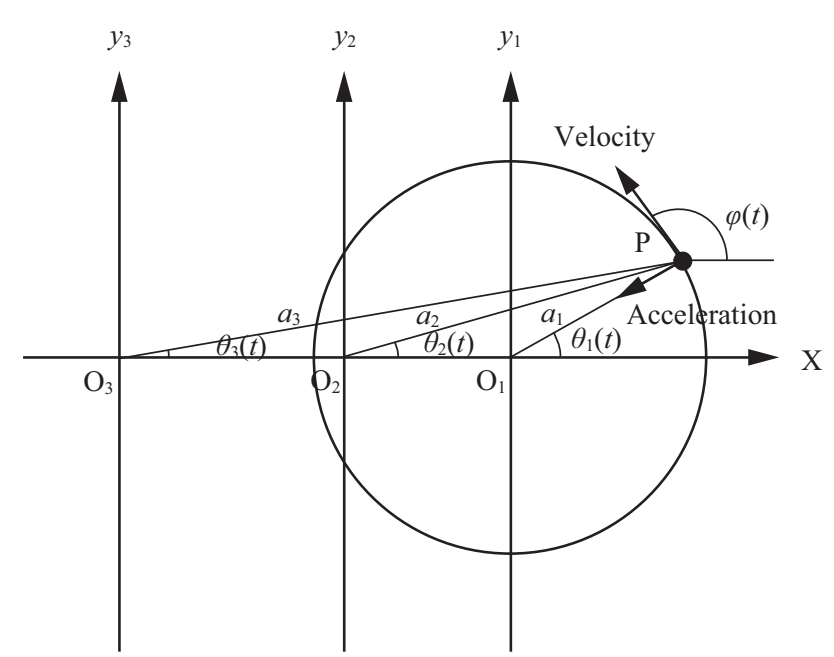

Fig. 4. The trajectory of particle, $P$, moving along a circle counterclockwise with constant angular speed. The correct radius of the circle is $R\left(a_{1}\right)$ (not $a_{2}$ or $a_{3}$ as calculated by Gabor's method). A circular type trajectory should be a "circle" in $x-y$ plane regardless the locations of the origin (global zero) $\mathrm{O}_{1}, \mathrm{O}_{2}$ or $\mathrm{O}_{3}$. For a circular hodograph, the corrected unwrapped phase should be $\theta_{1}(t)$ (not $\theta_{2}$ or $\left.\theta_{3}\right) . \theta_{1}(t)$ is equal to $\varphi(t)-\pi / 2$ which is the angle between $x$ axis and the tangential line at point $P$ (or velocity vector at point $P)$. Instantaneous amplitude can be calculated from the centripetal acceleration.

the OC method, the location of the origin (global mean) is ignored and the IF and IA are derived directly from the hodograph itself. Take previously discussed example: Imagine a particle, $\mathrm{P}$, traveling counterclockwise in a circular trajectory with a radius, $R$, and a constant angular speed, regardless whether the origin (global zero) is at $\mathrm{O}_{1}, \mathrm{O}_{2}$ or $\mathrm{O}_{3}$ in Fig. 4, the trajectory is always a "circle" in the $\mathrm{x}-\mathrm{y}$ plane. For a circular hodograph (Fig. 4), the corresponding unwrapped phase, $\theta_{1}(t)$, can be obtained by subtracting $\pi / 2$ from the angle $\varphi(t)$. This is the angle between $x$ axis and the tangential line at point $\mathrm{P}$ (or velocity vector at point $\mathrm{P}$ ). The IF is calculated as the derivative of unwrapped phase $\theta_{1}(t)$. Using this approach, let's define an osculating circle, $\mathrm{C}$, of a curve at a given point, $\mathrm{P}$, as the circle that has the same tangent and the same curvature as the curve at point $\mathrm{P}[5,9]$. Let's also define the inverse of the curvature as the radius of the osculating circle. With these definitions, the corresponding IA can be obtained from the inverse of the curvature of the osculating circle at point $\mathrm{P}$ (or centripetal acceleration vector at point $\mathrm{P}$ ). Therefore, at any point, $\mathrm{P}$, of the trajectory constructed by a time series itself and its HT, we can find a local inner tangent circle, the osculating circle, which has the same tangent as the trajectory at point $\mathrm{P}$ and the same curvature as the trajectory at point $\mathrm{P}$. The unwrapped phase $\theta(t)$ is derived from the tangential line of the trajectory at point $\mathrm{P}$, and IA is the radius of the osculating circle at point $\mathrm{P}$. Thus, procedure of the OC method for calculating IF and IA is:

Step 1: For a giving time series $x(t)$, apply HT to obtain the imaginary part, $y(t)$. These two time series form the trajectory of a particle.

Step 2: Calculate the velocity vector of the particle and then the unwrapped phase, $\theta(t)$, at time, $t$, as

$$
\theta(t)=\varphi(t)-\frac{\pi}{2}
$$

where $\varphi(t)$ is the angle between $x$ axis and the tangential line at point $\mathrm{P}$.

Step 3: Calculate $\operatorname{IF}(t)(5)$ in $\mathrm{Hz}$ as

$$
\mathrm{IF}(t)=\Omega(t)=\frac{1}{2 \pi} \frac{d \theta(t)}{d t}
$$

From [8], for a vector function, $\vec{r}(t)$, where $t$ is time, the curvature $\kappa(t)$ at point $\mathrm{P}$ can be calculated as

$$
\kappa(t)=\frac{\sqrt{\left(\vec { r } ^ { \prime } ( t ) \cdot \vec { r } ^ { \prime } ( t ) \left(\vec{r}^{\prime \prime}(t) \cdot \vec{r}^{\prime \prime}(t)-\left(\vec{r}^{\prime}(t) \cdot \vec{r}^{\prime \prime}(t)\right)^{2}\right.\right.}}{\left(\vec{r}^{\prime}(t) \cdot \vec{r}^{\prime}(t)\right)^{3 / 2}}
$$

where $\vec{r}^{\prime}(t)$ and $\vec{r}^{\prime \prime}(t)$ are first and second derivative of vector $\vec{r}(t)$.

Step 4: The amplitude, $\operatorname{IA}(t)$, that corresponding to this $\operatorname{IF}(t)$ can be found by

$$
\mathrm{IA}(t)=R(t)=\frac{1}{\kappa(t)}
$$

\section{CASE STUDY AND COMPARISON}

We will re-examine the example discussed in previous section using the new OC method.

\section{Simple Harmonic Function with Non-zero Mean}

In the case of a simple harmonic function with a non-zero mean (or DC offsets)

$$
x(t)=d+R \cos \omega t
$$

We have shown that when $|d|>R$, the traditional Gabor method will result negative IF from HT unwrapped phase-time plot. Using the OC method, the HT of (12) is

$$
y(t)=R \sin \omega t
$$

The trajectory, $\vec{r}(t)$, of particle, C, can be expressed as

$$
\vec{r}(t)=(d+R \cos \omega t) \vec{i}+(R \sin \omega t) \vec{j}
$$

The unit tangent vector of $\mathrm{C}$ is

$$
\vec{r}^{\prime}(t)=(-R \omega \sin \omega t) \vec{i}+(R \omega \cos \omega t) \vec{j}
$$


The unwrapped phase is

$$
\theta(t)=\tan ^{-1}\left(\frac{R \omega \cos \omega \mathrm{t}}{-R \omega \sin \omega \mathrm{t}}\right)-\frac{\pi}{2}=\omega t
$$

The IF is

$$
\Omega(t)=\frac{d \theta(t)}{d t}=\omega
$$

The centripetal acceleration of the particle $[8,9]$ is

$$
\kappa(t)=\frac{1}{R}
$$

The amplitude of instantaneous frequency (radius of the circle) at time $t$ is

$$
A(t)=\frac{1}{\kappa(t)}=R
$$

With this method, regardless the magnitude of the DC offset, $d=0,|d| \leq R$, or $|d|>R$, IF, and IA are constant (shown as the solid line in Fig. 3(d) and Fig. 3(f)). As we take the time derivative of the trajectory to calculate the velocity vector, the constant DC shift is dropped. In this example, the OC method correctly concluded that IF and IA are constant.

This OC method can be applied to any hodograph of phasor defined by a time series and its Hilbert Transform.

\section{Adding Two Simple Harmonic Signals Together}

For the combined simple harmonic signal example

$$
x(t)=\cos (a t / 2)+k \cos a t
$$

where $k=0.8$ and $a=2 \pi / 512$. The OC in this example would have a changing radius as the circle moves along the trajectory. The unwrapped phases can be calculated from the tangent line at any point of time. The dash lines in Fig. 2 are obtained from the OC method. The first three figures $(a, b$, and c) are the same for both the tradition Gabor's method and the OC method. The dash line in Fig. 2(d) is the unwrapped phase-time plot and the dash line in Fig. 2(e) is the IF given by (9). IA, the radius of osculating circles, which is equivalent to the derivative of the velocity for the particle trajectory at point $\mathrm{P}$ is given by (11) and showed as the dash line in Fig. 2(f). As shown in Fig. 2(d), the slope of unwrapped phase is always positive, so is the IF shown as the dash line in Fig. 2(e).

From Fig. 2(e) and 2(f), due to the larger radius of osculating circle at point $A$ than it is at point B on Fig. 2(c), the phase change is faster at point $B$. Thus, the frequency at point $\mathrm{B}$ is higher than at point $\mathrm{A}$. On the other hand, the radius of osculating circle at point A on Fig. 2(c) is smaller than the length of OA, thus the IA obtained by OC method is smaller than the IA from Gabor's method.

\section{Frequency Shift Cosine Waves}

For the frequency shift cosine waves

$$
\begin{array}{ll}
x(t)=\cos (a t) & 0 \leq t \leq 1023 \\
x(t)=\cos (a t / 2) & 1024 \leq t \leq 2047
\end{array}
$$

where $a=2 \pi / 128$. The frequency shift cosine wave $x(t)$ is showed in Fig. 5(a). Fig. 5(b) is the HT of $x(t)$. Fig. 5(c) is the trajectory of a particle formed by $x(t)$ and its HT. For a particle moving along the trajectory counterclockwise as shown in Fig. 5(c), the unwrapped phase-time plot in Fig. 5(d). The instantaneous frequency obtained by (8) and (9) is in Fig. 5(e) and the instantaneous amplitude is in Fig. 5(f). Fig. 6 is the Hilbert spectrum for the data on Fig. 5(a), using HHT for comparison [7].

Look at Fig. 5(e) and 5(f), the end effects manifested at the ends of the time series and at frequency shift region could be found. The end effects could not be prevented in this example due to limited data points in this time series. The spike at frequency shift region is due to an abrupt change of unwrapped phase. These problem could be solved or depressed by smoothing the unwrapped phase at adjacent points. Compare Fig. 5(e) and Fig. 6, both of them showing sharp frequency and time localizations, but in Fig. 6 the frequencies shift smoothly from high frequency to low frequency.

\section{DISCUSSIONS}

The OC method treats the input data (signal plus noise) as signal only. For real data with both signal and noise, the second derivative of vector $\vec{r}(t)$ may sharply fluctuate from time to time. Thus, the calculated curvature could be negative. In some cases, the original data is not varied smoothly, even the first derivative of vector $\vec{r}(t)$, could be negative as showed in previous example. Thus, when the input data are signal mixed with noises, when preprocesses are not applied to remove or depress the noise, the calculated $\operatorname{IF}(t)$ may quite different from the $\operatorname{IF}(t)$ obtained from signal only. One way to solve the problem, is removed the noise before applying the OC method, such as apply FFT or HHT to the time series first, then remove the highest frequency component. The other way is using moving average to smooth the unwrapped phase etc. before calculating IF and IA by using OC method.

For signal that comprises two wave components, there are six unknowns: two frequencies, two amplitudes and two phases. Theoretically, the IF and IA could be extracted by solving six nonlinear equations simultaneously. For data more than six points, these six unknown parameters could be solved by solving a series of six unknowns over determined nonlinear equations.

\section{CONCLUSIONS}

In this paper, the OC method, a new method of calculating 


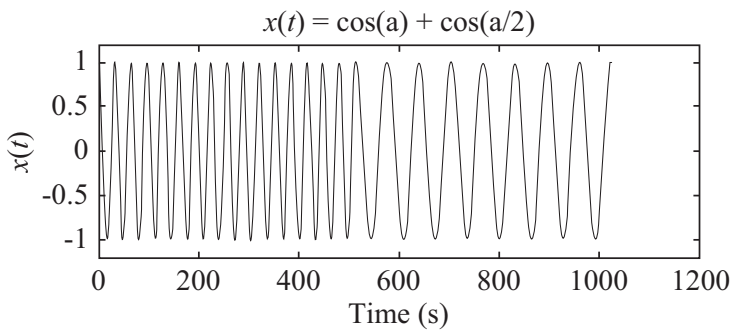

(a)

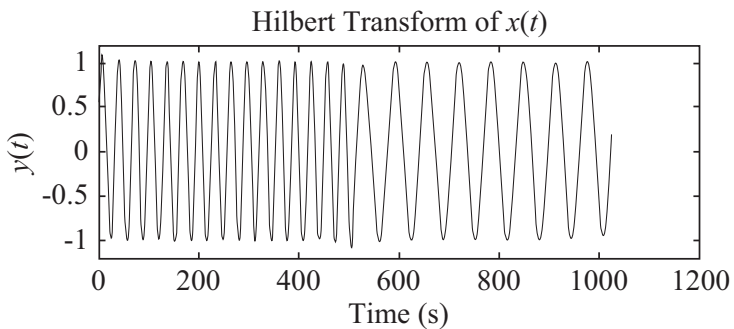

(b)

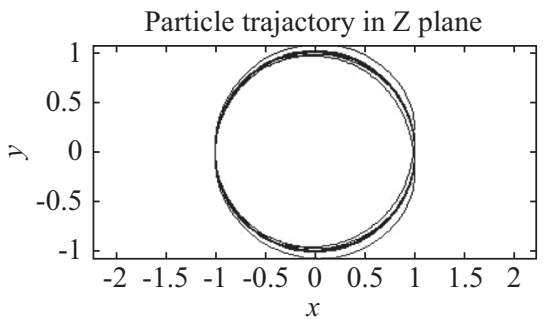

(c)

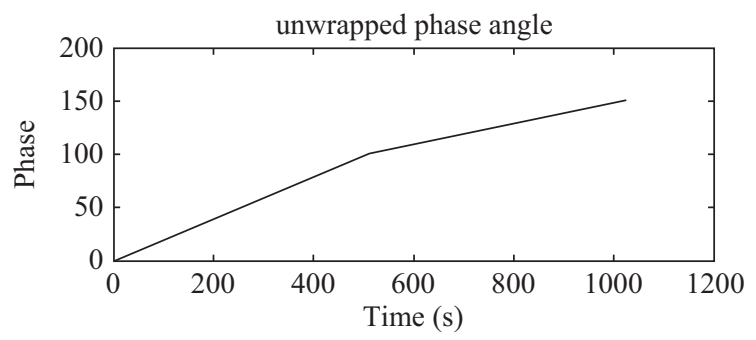

(d)

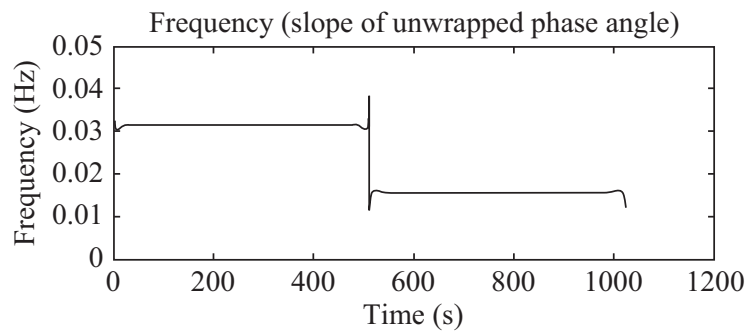

(e)

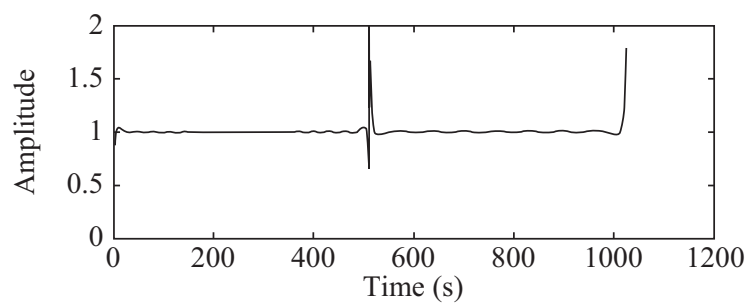

(f)

Fig. 5. (a) The frequency shift cosine wave $a=2 \pi / 128 x(t)=\cos a t, 0 \leq t \leq 1023, x(t)=\cos (a t / 2), 1024 \leq t \leq 2047$, (b) HT of $x(t)$, (c) the path of the particle, (d) unwrapped phase vs. time, (e) instantaneous frequency, and (f) instantaneous amplitude.

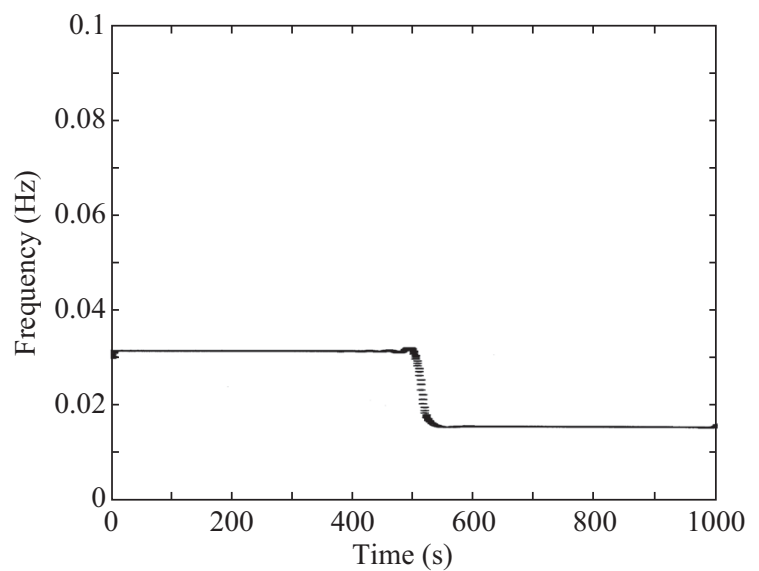

Fig. 6. The Hilbert spectrum for the data on Fig. 5(a), showing sharp frequency and time localizations. (From Huang, 1998)

IF and IA is introduced. This method employs the basic approach from Gabor's method with modified definitions of unwrapped phase, IF and IA. The OC method eliminated the possibility of arriving negative frequency results. Since the osculating circle of a given curve at any given time is unique, there will be only one corresponding IF and IA at any instant.
The OC method calculates the combined effects at any instant with no decomposition. This fact applies to any time series whether it is a simple harmonic function or a superposition of many linear components.

The new approach treats the input data as signal without any noise. If the input data is signal mixed with noises, directly use the OC method may cause unpredicted errors, when IF and IA are calculated. In order to get meaningful information, when apply OC method to real data, preprocess with care is necessary to decrease the effect of noise. Since, OC method provide a more accuracy IF and IA than the tradition Gabor's method. One potential application of OC method is applying OC method to estimate IF and IA for each IMFs obtained by EMD method [7].

\section{ACKNOWLEDGMENTS}

The first author would like to thank Academician N.E. Huang who introduced the EMD method when the first author visited NASA in 1998, and subsequently gave the first author many valuable comments on this new OC method. The first author also would like to thank Dr. Antony K. Liu for inviting the first author to conduct research at NASA in 1998 where the 
first author originated the concept of the OC method. The financial support from ONR and National Taiwan Ocean University (NTOU) that enabled the first author to visit NASA is greatly appreciated. The continuous support from National Science Council (for 2008 project no. is NSC 97-2611-M149-001), and support from Technology and Science Institute of Northern Taiwan (TSINT) is also greatly appreciated. TSINT has filed a patent application for the OC method.

\section{REFERENCES}

1. Boashash, B., "Estimating and interpreting the instantaneous frequency of a signal-Part 1: Fundamentals," Proceedings of the IEEE, Vol. 80, No. 4, pp. 520-537 (1992).

2. Boashash, B., "Estimating and interpreting the instantaneous frequency of a signal-Part 2: Algorithms and Applications," Proceedings of the IEEE, Vol. 80, No. 4, pp. 540-568 (1992).
3. Cohen, L., Time-Frequency Analysis, Prentice-Hall, Englewood Cliffs, NJ (1995).

4. Gabor, D., "Theory of communication," Proceedings of the Institute of Electrical Engineers, Vol. 93, pp. 429-457 (1946).

5. Gray, A., Modern Differential Geometry of Curves and Surfaces with Mathematica, 2nd Ed., CRC Press, Boca Raton, FL (1997).

6. Hahn, S. L., Hilbert Transforms in Signal Processing, Artech House, Inc., Boston (1996).

7. Huang, N. E., Shen, Z., Long, S. R., Wu, M. L., Shih, H. H., Zheng, Q., Yen, N.-C., Tung, C. C., and Liu, H. H., "The empirical mode decomposition and the Hilbert spectrum for nonlinear and non-stationary time series analysis," Proceedings of the Royal Society London, Series A, Vol. 454, pp. 903-995 (1998).

8. Kreyszig, E., Advanced Engineering Mathematics, 8th Ed., John Wiley \& Sons Inc., New York (1999).

9. Weisstein, E. W., Osculating Circle, From MathWorld-A Wolfram Web Resource, available: http://mathworld.wolfram.com/OsculatingCircle.html 